\title{
GAIA Level 3 Neonatal Invasive Bloodstream Infection
}

National Cancer Institute

\section{Source}

National Cancer Institute. GAIA Level 3 Neonatal Invasive Bloodstream Infection. NCI

Thesaurus. Code C127986.

GAIA Level 3 Neonatal Invasive Bloodstream Infection is defined by two criteria: first, the criteria does not meet the requirements for level 1 or 2 diagnostic certainty; second, two or more of the following requirements must be met: a) Temperature greater than or equal to 37.5 degrees $C$ or less than 35.5 degrees $C ;$ b) Tachypnea or severe chest indrawing or grunting or cyanosis; c) Change in level of activity; d) History of feeding difficulty; e) History of convulsions. 